Cahiers de recherches médiévales

\title{
Les opérations combinées des ordres militaires dans le Levant médiéval \\ (XII ${ }^{\mathrm{e}}$-XIII ${ }^{\mathrm{e}}$ siècles)
}

Pierre-Vincent Claverie

\section{(Q) OpenEdition \\ Journals}

\section{Édition électronique}

URL : https://journals.openedition.org/crm/5772

DOI : $10.4000 / \mathrm{crm} .5772$

ISSN : 1955-2424

\section{Éditeur}

Honoré Champion

\section{Édition imprimée}

Date de publication : 20 juin 2008

Pagination : 203-215

ISSN : 1272-9752

Référence électronique

Pierre-Vincent Claverie, «Les opérations combinées des ordres militaires dans le Levant médiéval 》, Cahiers de recherches médiévales [En ligne], 15 | 2008, mis en ligne le 20 juin 2011, consulté le 15 décembre 2022. URL : http://journals.openedition.org/crm/5772; DOl : https://doi.org/10.4000/crm. 5772 


\section{酷 $\mathrm{R}$}

\section{Les opérations combinées des ordres militaires dans le Levant médiéval (XII ${ }^{\mathrm{e}}$-XIII ${ }^{\mathrm{e}}$ siècles)}

L'historiographie des croisades a véhiculé depuis le XIX ${ }^{\mathrm{e}}$ siècle un nombre incalculable de poncifs sur le compte des ordres militaires, qu'elle a, selon les périodes, encensés ou voués aux gémonies. René Grousset s'est ainsi complu dans ses ouvrages sur la croisade à dénoncer «l'esprit impolitique» des templiers qui, auraient nourri durant près de deux siècles une haine inextinguible à l'encontre des hospitaliers'. Ces accusations excessives tirent leur origine du flot de critiques qui submergea l'Occident à l'annonce de la chute d'Acre en 1291. Plusieurs chroniqueurs répandirent, en effet, la rumeur que «si les frères des maisons de l'Hôpital, du Temple et de Sainte-Marie des Allemands et le reste des Acconitains avaient été en accord, la cité n'aurait jamais été prise ». Le pape Nicolas IV prit quelques distances avec ces reproches en qualifiant «d'athlètes du Christ et de combattants élus» les frères tombés en Syrie dans le récit qu'il donna des événements en août $1291^{2}$. Ces vues contradictoires méritent que l'on examine de plus près la collaboration développée par les ordres militaires dans le cadre des croisades. Plusieurs dizaines de lettres et de chroniques viennent compléter le témoignage des sources normatives sur la fraternité d'armes liant ces congrégations instituées au XII ${ }^{\mathrm{e}}$ siècle. L'article qui suit se contentera d'exposer quelques cas particuliers après avoir insisté sur les éléments de convergence livrés par les sources normatives.

\section{I- L'arrière-plan juridique : la leçon des règles et des statuts.}

Les règles édictées par les ordres de chevalerie recèlent de nombreuses informations sur leurs modes de coexistence en Orient. Une demi-douzaine d'articles de la règle du Temple traite ainsi du comportement à adopter en présence des hospitaliers, en consacrant une part belle aux combats : Ainsi un templier coupé des siens doit-il rallier la première bannière chrétienne venue, en choisissant de manière préférentielle celle de l'Hôpital. Les templiers consentaient, dans le même esprit, à ce qu'un frère hébergé à proximité d'un campement de l'Hôpital puisse abandonner sa tente sans encourir de poursuites disciplinaires. Les frères du Temple reçurent à la même époque l'autorisation de déguster du vin, à plus d'une lieue de leur commanderie, à l'invite de prélats ou d'hospitaliers connus pour leur modération. Mais le plus bel exemple connu de solidarité hospitalo-templière est fourni par le cas d'un commandeur templier, entré à Jérusalem en 1260 avec un

${ }^{1}$ R. Grousset, Histoire des croisades et du royaume franc de Jérusalem, Paris, 1936, t. III, p. 223.

${ }^{2}$ G.H. Pertz, Eberhardi archidiaconi Ratisbonensis annales, M.G.H., Scriptores, Hanovre, 1861, t. XVII, p.594; J. Heller, Iohannis de Thilrode Chronicon, M.G.H., Scriptores, Hanovre, 1880, t. XXV, p. 579.

Cahiers de Recherches Médiévales, 15, 2008 
contingent d'hospitaliers, qu'il se refusa d'abandonner dans la ville en état de siège lorsque l'ordre lui en fut donné. Lors du procès qui s'ensuivit, quatre de ses compagnons faillirent perdre leur habit pour avoir obtempéré à l'ordre du grand maître Thomas Bérard, sans attendre leur supérieur ${ }^{3}$. Malgré leur connivence réelle, les deux ordres proscrivirent très tôt la possibilité de transfert entre leurs couvents respectifs.

La même empathie unit longtemps les templiers aux chevaliers teutoniques, dont ils avaient parrainé la militarisation en 1198 en leur communicant un exemplaire de leur règle. Un passage de la règle du Temple de Barcelone témoigne des relations fusionnelles entretenues par certains templiers allemands avec leurs protégés durant la première moitié du XIII ${ }^{\mathrm{e}}$ siècle. Deux frères habilités à se rendre à Acre auraient fait amende honorable après avoir échangé leur habit avec des chevaliers teutoniques à l'occasion d'un repas trop arrosé. "Leur faute fut jugée sans perte d'habit, commente la règle, car les frères étaient jeunes et la chose faite simplement, les frères ayant récupéré leurs manteaux dans la journée $»^{4}$. La même bienveillance s'exerçait à l'égard de l'ordre de Saint-Lazare qui accueillait les templiers et hospitaliers atteints de lèpre. Ces transferts restaient toutefois subordonnés à l'aval de la hiérarchie après l'établissement formel du diagnostic par un médecin. Ce protocole ne dispensait pas les postulants de demander une confirmation de leur transfert auprès du Saint-Siège afin de régulariser leur situation sur le plan canonique. La règle de l'ordre de Saint-Lazare se fait l'écho de ces liens privilégiés en préconisant de consulter des prud'hommes du Temple et de l'Hôpital en cas de lacune jurisprudentielle ${ }^{5}$.

\section{II - L'organisation des opérations combinées au XII siècle}

Les ordres militaires prirent très tôt l'habitude de collaborer sur les champs de bataille de Palestine, que ce soit à Hārim (1164), à Montgisard (1177), à Margelion (1179), à La Fontaine du Cresson, et enfin à Hattīn (1187). Leur première opération combinée remonte au siège de Damas, conduit par les armées de la seconde croisade en 1148. Les chrétiens durent lever le camp, après quelques succès prometteurs, pour refluer précipitamment vers Jérusalem. Des rumeurs de trahison infondées accusèrent les templiers et les hospitaliers d'avoir perçu des fonds occultes de l'ennemi afin d'écourter les opérations. Les deux ordres participèrent, par la suite, au siège d'Ascalon qui vit une quarantaine de chevaliers du Temple trouver la mort aux côtés du grand maître, Bernard de Trémelay, en août 1153. Il fallut toute l'énergie du patriarche Foucher d'Angoulême et du maître de l'Hôpital, Raymond du Puy, pour convaincre le roi de Jérusalem de poursuivre le siège de la cité jusqu'à sa reddition. La même détermination permit en 1163 à un contingent de

\footnotetext{
${ }^{3}$ H. de Curzon, La règle du Temple, Paris, 1886, § 145 p. 114, 167-168 p. 126-127, 320 p. $186-187,421$ p. 230,429 p. 233 et 576-577 p. 299-300.

${ }^{4}$ J.M. Upton-Ward, The Catalan Rule of the Templars, Woodbridge, The Boydell Press, 2003, $\S 195$ p. 94.

${ }_{5}^{5}$ P.-V. Claverie, L'ordre du Temple en Terre sainte et à Chypre au XIII siècle, Nicosie, 2005 , t. II, p. $175-176$ et $177-178$.
} 
croisés angoumois de débloquer, avec l'appui du Temple, le château hospitalier du Crac des chevaliers, assiégé par l'atabeg de Damas, Nūr ad-Dīn (1146-1174).

La structure provinciale des ordres militaires facilita dès cette époque leur engagement concomitant sur plusieurs fronts. C'est ce qui advint en 1164 quand les couvents du Temple et de l'Hôpital accompagnèrent le roi Amaury de Jérusalem en Égypte, tandis que leurs garnisons antiochéniennes tentèrent de débloquer le château de Hārim, assiégé par Nūr ad-Dīn. La perte de 60 templiers antiochéniens poussa le grand maître Bertrand de Blanquefort à requérir l'assistance du roi de France, au mois de novembre, en évoquant pas moins de quatre théâtres d'opérations parallèles ${ }^{7}$. Son successeur Eudes de Saint-Amand contribua au succès de Montgisard, treize ans plus tard, à la tête d'un contingent de 80 templiers venus de Gaza en toute urgence. L'ost du royaume de Jérusalem dispersa, à cette occasion, la cavalerie de Saladin avec le soutien de l'Hôpital, dont le "palais des malades » de Jérusalem accueillit 750 blessés après la bataille ${ }^{8}$. Le grand maître Roger des Moulins diffusa la nouvelle que 30000 musulmans avaient succombé sous les coups des chrétiens, tandis que les malades de l'Hôpital attendaient, en prière, l'issue du combat. Cette relation empreinte d'exagération estime à 15000 le nombre de prisonniers faits dans la journée du 25 novembre 1177, en évaluant les pertes chrétiennes à 1100 hommes $^{9}$. Les ordres militaires participèrent avec moins de bonheur à la bataille de Margelion, engagée en juin 1179 au sud de la plaine de la Beqaa. Le Temple perdit dans cette chevauchée effrénée son maître et son sénéchal aux côtés d'un nombre appréciable de frères ${ }^{10}$.

La fraternité d'armes régnant entre les templiers et les hospitaliers ressort aussi de la bataille de La Fontaine du Cresson du premier mai 1187, où les grands maîtres Gérard de Ridefort et Roger des Moulins combattirent côte à côte. Gérard de Ridefort n'écouta pas les conseils de prudence du maître de l'Hôpital en décidant d'engager un combat inégal contre une colonne de 7000 musulmans sur le point de regagner ses bases. Roger des Moulins fut tué ainsi qu'une soixantaine de templiers. Parmi ces derniers se trouvaient la majeure partie de l'état-major du Temple et l'inestimable Jaquelin de Maillé, un templier de Touraine considéré comme l'un des meilleurs chevaliers du siècle. Gérard de Ridefort échappa aux sabres ayyoubides en fuyant piteusement, comme son subordonné l'avait prophétisé à l'orée du combat. Ce même grand maître provoqua la chute de la Terre sainte franque, quelques semaines plus tard, en incitant Guy de Lusignan à secourir inconsidérément la ville de Tibériade, assiégée par les musulmans. La bataille de Hattīn fut fatale aux Latins qui perdirent 230 templiers, décapités sur ordre de Saladin en compagnie de 120 chevaliers de 1'Hôpital. En outre, 150 de ces combattants semblent avoir trouvé la

${ }^{6}$ Guillaume de Tyr, Chronicon, éd. R.B.C. Huygens, Turnhout, 1986, t. II, XVII (4-7), p. $764-$ 776, XVII (27), p. 797-799 et XIX (8), p. 873-874 et J. Richard, Histoire des croisades, Paris, Fayard, 1996, p. 178-179, 186 et 190.

${ }^{7}$ J.-P. Migne, Patrologia latina, Paris, 1880, t. CLV, n 23 col. 1279-1280 et nº 13 col. $1272-$ 1273 ; Guillaume de Tyr, op. cit., t. II, XX (19), p. 936-937.

${ }^{8}$ Guillaume de Tyr, op. cit., t. II, XXI (21), p. 990-991.

${ }^{9}$ R. Röhricht, Beiträge zur Geschichte der Kreuzzüge, Berlin, 1878, t. II, p. 127-128

${ }^{10}$ Guillaume de Tyr, op. cit., t. II, XXI (28), p. 1001-1002; A. D'Albon, «La mort d'Odon de Saint-Amand », Revue de l'Orient latin, 12, 1909-1911, p. 280-282. 
mort à Damas où l'émir Safî ad-Dīn ibn al- 'Abid reçut l'ordre d'exécuter tous ceux qui lui tomberaient entre les mains ${ }^{11}$.

La défaite de Hattīn ébranla la structure des ordres combattants qui constituaient les deux tiers de la chevalerie du royaume de Jérusalem. Des partenariats locaux contribuèrent à assurer la survie de points de résistance comme la ville de Tyr, défendue par le marquis Conrad de Montferrat. Les troupes du templier Geoffray Morin et du grand-commandeur de l'Hôpital, Borrell, parvinrent à capturer, le 30 décembre 1187, une escadre de onze galères ayyoubides participant au blocus maritime de la cité. Les chrétiens armèrent, à cette occasion, une dizaine d'embarcations légères aux côtés d'une escadre de 17 galères. Cette intervention illustre les collaborations fructueuses que les ordres militaires pouvaient développer localement avec le concours des laïcs. Elle les familiarisa au combat sur mer qui devait mobiliser une part croissante de leurs effectifs au XIII ${ }^{\mathrm{e}}$ siècle. La résistance de Tyr permit au roi Guy de Lusignan d'entreprendre le siège d'Acre, en 1189, avec une armée de 9000 hommes, soutenue par le Temple et l'Hôpital. ${ }^{12}$.

Alain Demurger a souligné, il y a quelques années, la difficulté à évaluer les contingents des ordres militaires associés aux opérations de la troisième croisade. Selon lui, le Temple et l'Hôpital paraissent avoir fourni un sixième des combattants impliqués dans la victoire d'Arsur du 7 septembre 1191. Un libelle composé au lendemain de la défaite de Hattīn nous éclaire sur la spécialisation des ordres militaires dans l'encadrement des armées occidentales opérant au Levant. Les templiers constituaient à Hattīn l'arrière-garde de l'ost rassemblé par Guy de Lusignan afin de débloquer la citadelle de Tibériade. Deux lettres de la fin du mois de septembre 1187 célèbrent leur comportement héroïque lors de la bataille du 4 juillet, dont ils ont remporté les premiers assauts comme de «véritables lions $»^{13}$. Cette situation aurait conduit Saladin à les attaquer en personne afin de doper le moral de ses troupes à en croire plusieurs chroniqueurs orientaux ${ }^{14}$. La solidarité régnant en 1187 entre les templiers et les hospitaliers est à l'origine de nombreuses confusions dans le récit des événements dressé par les historiens arabes. La palme de l'admiration revient incontestablement au jacobite Grégoire Abū'l Faradj, qui estime à 500 dinars le prix moyen des chevaliers du Temple rachetés par Saladin, le surlendemain de sa victoire, afin de les exécuter ${ }^{15}$.

${ }^{11}$ P.-V. Claverie, «Les débuts de l'ordre du Temple en Orient », Le Moyen Age, 111, 2005, p. 582-583 et A. Demurger, "Templiers et Hospitaliers dans les combats de Terre Sainte», Actes du XVIII congrès des historiens médiévistes de l'Enseignement supérieur public, Montpellier, 1987 , p. 83 et $79-80$ pour une comparaison des sources narratives et diplomatiques, que nous avons systématiquement privilégiées pour notre part.

${ }^{12} \mathrm{G}$. Müller, Documenti sulle relazion delle città toscane coll'Oriente, prem. part., Florence, $1879, n^{\circ} 23-25$ p. 26-31; M. Salloch, Die lateinische Fortsetzung Wilhelms von Tyrus, Greifswald, 1934, $\mathrm{n}^{\circ}$ VII p. 87-88; A. Demurger, art. cit., p. 81.

${ }^{13}$ R. Röhricht, Regesta Regni Hierosolymitani, Innsbruck, 1904, t. II, n 664, p. 45-46 et t. I, $1893, n^{\circ} 661$, p. 176

${ }^{14}$ Būtros Ibn Ar-Rāhib, Chronicon Orientale, tr. L. Cheikho, Beyrouth-Paris, 1903, t. II, p. 99 ; Michel le Syrien, Chronique, éd. J.-B. Chabot, Paris, 1905, t. III, p. 404.

${ }_{15}$ Abū'l Faradj, The Chronography, éd. E.A. Wallis Budge, Oxford-Londres, 1932, t. I, p. 324-325. 
Deux sources de la troisième croisade permettent de suivre l'activité des ordres durant la décennie suivante : Il s'agit de l'Estoire de la guerre sainte du poète Ambroise et de l'Itinerarium peregrinorum, dont la trame fut consignée en 1192 par le chapelain anglais Richard du Temple. Elles révèlent le cantonnement des templiers et des johannites dans le faubourg de Montmusard durant le long siège d'Acre de 1189-1191. Les «preux seigneurs du Temple » commencèrent par assurer l'arrière-garde de l'ost de la croisade en novembre 1190, avant de troquer leur place avec les hospitaliers le 27 août 1191. Les templiers assurèrent à nouveau l'arrièregarde de l'armée quand elle prit la direction de Césarée, après une halte de quelques jours dans le casal de Merle. Les frères du Temple perdirent un nombre incalculable de montures dans les accrochages qui scandèrent la progression des croisés vers Arsur. Ils retrouvèrent leur position initiale au moment de la bataille du 7 septembre, après une nouvelle permutation avec l'Hôpital, qui reçut, pour la circonstance, le soutien de hauts barons. Les templiers glissèrent à nouveau à l'arrière de l'ost quand l'armée prit la route de Jaffa après trois jours de récupération ${ }^{16}$. Ce rôle devait tellement se généraliser au cours du XIII ${ }^{\mathrm{e}}$ siècle, que Jacques de Molay en fit un argument de poids lorsqu'il dut défendre la spécificité de son ordre devant la papauté17.

La troisième croisade associa le Temple et l'Hôpital au sein de nombreuses opérations diplomatiques ou tactiques. Les archers montés des deux ordres se firent une spécialité de parcourir les champs de bataille du Levant afin de relever les corps des croisés tués au combat. Les maîtres des deux maisons dissuadèrent les croisés restés en Orient après le départ de Philippe Auguste de marcher contre Jérusalem sans un important soutien tactique. Ils organisèrent au mois de mai 1192 une chevauchée dans la région de Darom, qui se solda par la capture d'une vingtaine de musulmans. Les deux ordres repoussèrent, le 12 juin suivant, un parti de 200 musulmans informés de la progression du roi d'Angleterre en direction de la fontaine d'Emmaüs. Les hommes de Robert de Sablé et Garnier de Naplouse - à la tête du Temple et de l'Hôpital - travaillèrent en parfaite intelligence, au mois de juillet 1192, au démantèlement des places de Darom et d'Ascalon, reprises par les croisés. Les deux maisons soutenaient, à cette date, les pourparlers engagés par le roi d'Angleterre avec Saladin à partir d'Acre, puis de Césarée à la suite d'un raid ayyoubide contre Jaffa. Leur soutien sans faille aboutit le 31 août 1192 à la signature d'une trêve triennale, qui stabilisa de fait la situation politique de la région pour près de quatre décennies ${ }^{18}$. Cette attitude des ordres a conduit Joseph Delaville Le Roulx à affirmer très tôt que « sans eux, Richard n'eût rien pu entreprendre en Syrie ${ }^{19}$.

\footnotetext{
${ }^{16}$ Ambroise, The History of the Holy War. Estoire de la Guerre Sainte, éd. M. Ailes et M. Barber, Woodbridge, 2003, t. I, p. 65 , v. 4039, p. 95, v. 5858, p. 96, v. 5942-5943, p. 98 , v. 6042 , p. 99 , v. 6140 , p. 112 , v. $6902-6903$ et p. 117 , v. $7225-7226$, Itinerarium peregrinorum et gesta regis Ricardi, éd. W. Stubbs, Chronicles and Memorials of the Reign of Richard I, Londres, 1864, t. I, p. 253, 255, 260, 265 et 281

${ }^{17}$ A. Demurger, art. cit., p. 91.

${ }^{18}$ Itinerarium peregrinorum et gesta regis Ricardi, éd. W. Stubbs, op. cit., t. I, p. 276, 308, 346, 371, 399, 404-405.

19 J. Delaville Le Roulx, Les Hospitaliers en Terre sainte et à Chypre (1100-1310), Paris, 1904, p. 115
} 


\section{III - La perpétuation du modèle durant le siècle de saint Louis}

Le XIII ${ }^{\mathrm{e}}$ siècle vit l'entente des ordres militaires se renforcer malgré la multiplication des sujets de contentieux au niveau territoria ${ }^{20}$. Le cadre politique de l'Orient latin subit de nombreux changements jusqu'à la chute finale de la Terre sainte en 1291. Les ordres du Temple et de l'Hôpital reçurent dans ce contexte troublé le soutien de nouvelles congrégations combattantes, comme l'ordre teutonique ou celui de Saint-Lazare. Ils participèrent dès 1203 aux chevauchées que le roi Aimery de Lusignan effectua en Palestine avec le concours de chevaliers occidentau $^{21}$. De même, durant la cinquième croisade les teutoniques épaulèrent les templiers et les hospitaliers dans le delta du Nil. Templiers et teutoniques repoussèrent à ce titre, le 31 juillet 1219 , une violente offensive ayyoubide contre les lices chrétiennes. Leur victoire inespérée souleva l'admiration de nombreux chroniqueurs, prompts à célébrer, à l'instar de l'écolâtre Olivier le Scolastique, « le courage des templiers et de la maison des Allemands $\iota^{22}$. Le Temple sauva à nouveau l'ost d'une déroute annoncée, un mois plus tard, après la débandade d'un corps d'armée romain remontant le Nil: Les hommes de Guillaume de Chartres perdirent dans l'affrontement une trentaine de chevaliers aux côtés de 60 à 70 sergents à cheval et turcoples, c'est-à-dire archers montés ${ }^{23}$.

Plusieurs chroniques affirment que les ordres fournirent le 29 août 1219 de nombreux compagnons à saint Jean Baptiste après la décollation d'une cinquantaine de leurs hommes par les Égyptiens. Olivier le Scolastique signale au milieu de ces nouveaux «Roland» les templiers et les teutoniques, à la pointe de l'attaque durant toute la journée ${ }^{24}$. Très peu d'entre eux auraient fui, l'Hôpital enregistrant la perte d'une douzaine de combattants aux côtés de son maréchal Aymar de Layron ${ }^{25}$. Un récit contemporain souligne avec justesse que tous les chrétiens auraient été occis ou pris ce jour-là sans le dévouement du roi de Jérusalem et des ordres militaires. Les templiers gagnèrent l'admiration de nombreux chroniqueurs allemands, conscients des limites tactiques de l'ordre teutonique. Ces lacunes sautèrent aux yeux de tous lors du retour d'un commando templier d'un raid contre le fort de Burlus au mois de juillet 1220. Les éclaireurs turcs qui talonnaient les templiers capturèrent le maréchal ainsi que le grand commandeur de l'ordre teutonique, venus sans la moindre couverture à leur rencontre. Cette négligence n'empêcha pas les frères allemands de s'illustrer jusqu'au désastre de Barāmūn, en 1221, qui sonna le glas des ambitions latines en Égypte ${ }^{26}$.

\footnotetext{
${ }^{20}$ P.-V. Claverie, op. cit. en note 5, t. II, p. 51-99.

${ }^{21}$ Ernoul, Chronique, éd. L. de Mas Latrie, Paris, 1871, p. 354-356.

${ }^{22}$ Olivier le Scolastique, Historia Damiatina, éd. O. Hoogeweg, Die Schriften des kölner Domscholasters, späteren Bischofs von Paderborn und Kardinal-bischofs von S. Sabina, Oliverus, Tübingen, 1894, p. 210-211.

${ }^{23}$ P.-V. Claverie, op. cit., t. II, p. 61-62.

${ }^{24}$ Olivier le Scolastique, op. cit., p. 216-217 et 252-253.

${ }^{25}$ J. Delaville Le Roulx, op. cit., p. 144 et 410 à propos de la nomination du successeur d'Aymar, Féraud de Barras.

${ }^{26}$ R. Röhricht, Quinti Belli Sacri scriptores minores, Genève, 1879, p. 52, 102-103, 132-133 et 190-191.
} 
On assista au XIII ${ }^{\mathrm{e}}$ siècle à une multiplication des engagements contre les différentes principautés ayyoubides, qui avaient succédé en 1193 à l'empire unitaire de Saladin. Un corps d'armée hospitalo-templier fut taillé en pièces, le 16 mai 1203, par l'émir al-Mansūr I ${ }^{\text {er }}$ de Hama dans l'est du comté de Tripoli. Le maître du Temple, Philippe du Plessis, tenta de négocier la signature d'une trêve avec l'émir en faisant valoir le débarquement fictif de quelque 70000 croisés à Acre. Ce type d'intervention était subordonné à l'adhésion des ordres militaires aux mêmes stratégies politiques à une époque d'effacement, pour ne pas dire de vacance, du pouvoir royal. La moindre division ou dissension était alors susceptible de remettre en question la survie même de la Syrie latine. Ainsi, à l'expiration des trêves signées en 1204 par le royaume de Jérusalem avec l'Égypte, les templiers se retrouvèrent en conflit avec une partie de la noblesse franque en matière stratégique. Leurs aspirations bellicistes finirent cependant par triompher du projet de paix défendu par la majeure partie des « Poulains », des hospitaliers et des teutoniques ${ }^{27}$.

Dans le comté de Tripoli, où les motifs de querelle étaient nombreux, la collaboration des frères du Temple et de l'Hôpital ne s'interrompit jamais. On vit ainsi les deux ordres mobiliser plus de 3000 hommes, en 1230, afin d'imposer un tribut à l'émir de Hama dont les possessions jouxtaient leurs domaines. Ils subirent une importante défaite, au mois d'août, qui repoussa à l'hiver suivant l'organisation d'un raid contre la ville côtière de Gibel. Les deux ordres essuyèrent une vigoureuse contre-attaque de la part des forces alépines du côté de la cité de Valénie dont la citadelle fut pillée. Les templiers infligèrent, en retour, durant le printemps 1231 une sévère défaite à l'émir Badr ad-Dīn al-Malī, qui dut appeler des renforts. Le Temple et l'Hôpital profitèrent de ce succès pour signer avec les musulmans, au mois de juin, une trêve prévoyant un échange de prisonniers ${ }^{28}$.

Le comté de Tripoli offrit jusque dans les années 1260 un cadre privilégié de collaboration aux deux ordres, qui prélevaient conjointement un tribut sur la secte des Assassins implantée dans le Djebel Ansarieh. Templiers et hospitaliers avaient participé à la campagne de représailles menée par le prince Bohémond IV d'Antioche après l'assassinat de son fils Raymond dans la cathédrale de Tortose, en 1213. Ils organisèrent en 1233 une nouvelle campagne contre l'émirat de Hama, qui se dérobait au tribut imposé par l'Hôpital en 1229. Deux cents chevaliers laïques s'associèrent à cette expédition conduite par les maîtres du Temple et de l'Hôpital. La coalition chrétienne opéra sa concentration dans la plaine de La Boquée, au sud du Crac, avant de prendre la direction de Montferrand ou Ba'rīn. Une campagne d'une huitaine de jours lui permit de piller plusieurs cantons voisins, sans parvenir à reprendre aux musulmans la citadelle de Montferrand. L'arrivée inopinée du sultan d'Égypte al-Kāmil faillit, d'ailleurs, compromettre l'issue de la campagne. Le sultan

\footnotetext{
${ }^{27}$ E. Blochet, Histoire d'Égypte, dans Revue de l'Orient latin, 9, 1902, p. 126-127 [222-223].

${ }^{28} \mathrm{Ibn}$ al-Athīr, Extrait de la chronique intitulée Kamel-Altevarykh, Recueil des historiens des croisades, Historiens orientaux, Paris, 1887, t. II, p. 180 ; E. Blochet, Histoire d'Égypte, Revue de l'Orient latin, 10, 1903-1904, n. 3 p. 257 ; Aubry de Trois-Fontaines, Chronica, éd. P. Scheffer-Boichorst, M.G.H. Scriptores, Hanovre, 1874, t. XXIII, p. 928.
} 
invita heureusement son neveu al-Muzaffar à verser aux hospitaliers ses arriérés afin de pouvoir gagner au plus vite l'Euphratèse à la tête de son armée ${ }^{29}$.

La complicité liant les templiers aux hospitaliers ne fit pas toujours que des heureux. Le prince Bohémond V d'Antioche accusa ainsi les deux ordres, en 1236, de protéger les Assassins du Djebel Ansarieh, auxquels ils avaient imposé leur tutelle au XII ${ }^{\mathrm{e}}$ siècle; le prince se plaignait alors du désengagement des templiers d'une campagne militaire en Petite-Arménie, qu'il entendait prolonger pour des motifs personnels ${ }^{30}$. Les garnisons des deux ordres continuèrent durant la deuxième moitié du XIII ${ }^{\mathrm{e}}$ siècle à collaborer activement sur les terrains tactiques et diplomatiques. Les templiers dépêchèrent à ce titre, en 1254, une mission de secours vers la citadelle - mal en point - de Château-Blanc avec le concours des hospitaliers et du comte Bohémond V. La déroute des mamelouks fit l'objet d'une publicité jusque sur la frontière écossaise, où les moines de Lanercost célébrèrent l'unité des ordres $^{31}$. Les templiers rendirent la pareille aux johannites en expédiant, en 1266, au Crac des chevaliers une cinquantaine d'arbalétriers et d'archers. L'escouade fut anéantie impitoyablement par l'émir Sayf ad-Dīn Qalāwūn al-Alfì qui venait de détruire le château de Coliath, situé entre Tripoli et le Crac. Les templiers répondirent à la décapitation de leurs archers par un raid infructueux contre le campement de la cavalerie mamelouke. Ils expédièrent, après cette défaite, un espion sur les talons des mamelouks dans l'espoir de préparer une embuscade. Le malheureux fut démasqué et pendu, sans que les ordres militaires ne puissent récupérer aucune des positions enlevées par les mamelouks les jours précédents ${ }^{32}$.

Le royaume de Jérusalem concentra jusqu'en 1291 l'essentiel des effectifs des ordres combattants du fait de l'établissement de leurs couvents centraux à Acre. La réoccupation temporaire de la Ville sainte ne remit pas en cause ce principe entre 1229 et 1244 . Les ordres militaires s'ingénièrent à réfréner durant cette période l'enthousiasme des croisés qui débarquaient au Levant sans la moindre connaissance de la situation politique. Les maîtres du Temple et de l'Hôpital tentèrent ainsi de dissuader, le 12 novembre 1239, le duc de Bourgogne de mener une expédition contre un campement de Turcs, établi à proximité de Gaza. Les croisés se firent cependant tailler en pièces dans les dunes de Bayt Hānūn. Le poète Philippe de Nanteuil accusa le Temple et l'Hôpital dans une complainte cinglante d'un semblant de trahison, qu'un pamphlétaire local se fit un devoir de démentir ${ }^{33}$. Ces tensions pouvaient aussi affecter les ordres militaires en période d'hostilités. C'est ce qui advint en 1247 au sein de la garnison hospitalo-templière d'Ascalon, soumise à un

\footnotetext{
${ }^{29}$ Estoire d'Eracles, Recueil des historiens des croisades, Historiens occidentaux, Paris, 1859, t. II, p. 403-405 ; E. Blochet, op. cit., dans Revue de l'Orient latin, 10, 1903-1904, not. 2 p. 265 et p. 304.

${ }^{30}$ P.-V. Claverie, op. cit. en note 5, t. II, p. 139-140.

${ }^{31}$ Chronicon de Lanercost, MCCI-MCCCXLVI, éd. J. Stevenson, Edimbourg, 1839, p. 60

${ }^{32}$ P.-V. Claverie, op. cit. en note 5, t. II, p. 19 citant U. \& M.C. LyONS, Ayyubids, Mamlukes and Crusaders, Selections from the Tārīkh al-Duwal wa'l-Mulūk of Ibn al-Furāt, t. II, Cambridge, 1971, p. 85-86.

${ }^{33}$ Estoire d'Eracles, op. cit., p. $539-540$ et p. 549.
} 
blocus à la fois naval et terrestre. Ses trois commandeurs se résolurent à capituler après la destruction de la seconde enceinte du château par les forces égyptiennes ${ }^{34}$.

L'équilibre des forces entre musulmans et chrétiens avait basculé, trois ans plus tôt, lors d'un engagement militaire survenu dans la région de Gaza. La bataille de La Forbie mit aux prises une coalition franco-syrienne, rassemblant les émirs d'al-Karak et d'Homs, à une alliance de circonstance entre les Ayyoubides du Caire et un groupe de tribus turques, qui venaient de piller Jérusalem. Une série de lettres échangées avec l'Occident permet d'évaluer les contingents du Temple lors de cet affrontement à 312 chevaliers et 324 turcoples, contre 328 chevaliers pour l'Hôpital et 200 turcoples. Cette cavalerie semble avoir reçu le soutien de 400 chevaliers teutoniques et 600 chevaliers des royaumes de Chypre et de Jérusalem au sein d'une force globale de 16000 hommes. Les Francs occupaient l'aile droite du front, qui s'étendait en bordure de mer par déférence pour leurs alliés musulmans. Ce choix se révéla catastrophique lorsque la cavalerie égyptienne désintégra le centre et l'aile gauche de la coalition franco-syrienne, particulièrement friables ${ }^{35}$.

Les Francs tentèrent d'échapper à l'enveloppement qui les menaçait, sans parvenir à déployer leurs « échelles » correctement. Il s'ensuivit une mêlée confuse au cours de laquelle les Turcs capturèrent les grands maîtres du Temple et de l'Hôpital. Le maître des teutoniques, Gerhard von Malberg, parvint à s'enfuir avec 33 templiers, 26 hospitaliers et 3 teutoniques selon une lettre du patriarche de Jérusalem de la fin du mois de novembre 1244. Gerhard fut accusé de pusillanimité sur le champ de bataille et contraint de démissionner. Il quitta l'ordre pour rejoindre les rangs du Temple, selon toute évidence, au début de l'année suivante. Son confrère Guillaume de Châteauneuf dut attendre l'année 1250 pour reprendre les rênes de l'Hôpital ${ }^{36}$. Il fit partie du petit groupe de captifs de La Forbie qui dut sa libération à la ténacité manifestée par saint Louis durant les négociations qui suivirent sa défaite en Égypte. Guillaume de Châteauneuf regagna Acre en compagnie de 25 johannites, 15 templiers et 10 teutoniques $^{37}$.

L'engagement des ordres militaires fut total lors de la septième croisade. Le Temple semble avoir perdu à lui seul 280 hommes dans la journée du 7 février 1250 , qui vit sa cavalerie décimée dans les rues de la ville d'al-Mansūra. La responsabilité de cette défaite incombe pleinement au comte d'Artois, qui refusa d'obtempérer par deux fois à un ordre de repli adressé par son frère saint Louis. L'obstination du

\footnotetext{
${ }^{34}$ C. Cahen, «Une source pour l'histoire des croisades : les Mémoires de Sa'd ad-dīn ibn Hamawiya Juwaïnī», Bulletin de la Faculté des Lettres de Strasbourg, novembre 1949, p. 330 (repris dans Orient et Occident au temps des Croisades, Paris, 1992 ( $2^{\mathrm{e}}$ éd.), $\mathrm{n}^{\circ}$ XVIII p. 241).

${ }^{35}$ A. Demurger, art. cit en note 11, p. 80.

${ }^{36}$ M. L. Bulst-Thiele, «Zur Geschichte der Ritterorden und des Königreichs Jerusalem im 13. Jahrhundert bis zur Schlacht bei la Forbie am 17. Oktober $1244 »$, Deutsches Archiv für Erforschung des Mittelalters Namens der «Monumenta Germaniae historica», 22, 1966, p. 224-226.

${ }^{37}$ P.-V. Claverie, op. cit. en note 5, t. II, p. 109.
} 
comte n'est pas sans rappeler dans son inconséquence l'aveuglement des croisés bourguignons capturés dans les sables de Gaza en $1239^{38}$.

Dans les années qui suivirent les conditions politiques influèrent de plus en plus directement sur les campagnes menées le long du littoral syrien. Le gouverneur turc de Jérusalem captura ainsi, au début de l'année 1256, une centaine de chevaliers du Temple et de l'Hôpital, stationnés sur la frontière du comté de Jaffa. Ce fidèle serviteur du sultan de Damas, an-Nāsir Yūsuf, entendait répliquer à une chevauchée audacieuse de Geoffroy de Sergines, qui, dans les derniers jours de 1255, avait capturé ou tué quelque 1200 musulmans. Le chevalier bourguignon avait pris prétexte du fait que la seigneurie de Jaffa était restée en dehors de la trêve décennale conclue par les royaumes de Jérusalem et Damas, quelques mois plus tôt, pour lancer ce raid ${ }^{39}$. Le gouverneur de Jérusalem ne s'embarrassa pas de ces précautions lorsqu'il déroba aux ordres militaires 4000 têtes de bétail, disséminées le long de la frontière. Les templiers et les hospitaliers furent si éprouvés par cette déconvenue qu'ils entérinèrent la trêve de dix ans, signée peu après par les chrétiens avec les sultanats du Caire et de Damas ${ }^{40}$.

Ce n'est qu'après l'arrivée au pouvoir du sultan Baybars en 1260 que les ordres échafaudèrent à nouveau de pareilles initiatives. Ils entreprirent au mois de janvier 1264 une expédition contre la bourgade galiléenne d'al-Lajjûn, d'où ils ramenèrent 300 captifs et un nombreux cheptel. Ce succès les encouragea à organiser au mois de juin suivant une chevauchée dans la région de Jaffa et d'Ascalon en réponse à la capture infamante du châtelain de l'endroit. Ils défirent avec l'appui de Geoffroy de Sergines une halqa de 300 à 400 Turcs, avant de planifier au mois de novembre un raid plus ambitieux contre la ville de Bethsan. Les ordres militaires reçurent, pour ce faire, le soutien du croisé Olivier de Termes, nouvellement arrivé, et de la chevalerie séculière d'Acre. Cette force disparate incendia trois villages des environs de Bethsan où elle captura un nombre important $\mathrm{d}^{\prime}$ hommes et d'animaux ${ }^{41}$. Ces succès encouragèrent les templiers à lancer, en 1266 , une expédition contre la région de Tibériade en concertation avec le bayle de Chypre, Hugues de Lusignan. Une embuscade tendue le 28 octobre à hauteur de Tell Harūba décima l'avant-garde latine refluant vers Acre. Cette erreur imputable aux états-majors de l'Hôpital et de l'ordre teutonique coûta la vie à 500 hommes, que les fellahs des environs achevèrent pendant la nuit. On peut s'étonner néanmoins que le Temple ou la chevalerie chypriote ne soient pas parvenus à récupérer ces malheureux avant la tombée du jour à une si faible distance d'Acre ${ }^{42}$.

\footnotetext{
${ }^{38}$ Matthieu Paris, Chronica maiora, éd. H.R. Luard, Londres, 1872, t. IV, p. 337-344 ; P.-V. Claverie, op. cit., t. II, p. 71-73.

${ }^{39}$ C.J. Marshall, «The French regiment in the Latin East, 1254-91 », Journal of Medieval History, 15, 1989, p. 301-307.

${ }^{40}$ Estoire d'Eracles, op. cit., p. 630-631.

${ }^{41}$ R. Röhricht, «Annales de Terre Sainte », dans Archives de l'Orient latin, Paris, 1884, t. II B, col. B p. 451-452; R. de Mas Latrie, Chroniques d'Amadi et de Strambaldi, Paris, 1891, t. I, p. 206-207.

${ }^{42}$ Templier de Tyr, Cronaca del Templare di Tiro (1243-1314), éd. L. Minervini, Naples, 2000, 1113 (349), p. 112 ; R. Röhricht, art. cit., col. A et B p. 451-454.
} 
La convergence de vue régnant entre les ordres militaires se manifesta lors du passage en Orient d'un demi-millier de chevaliers catalans à l'automne 1269. Il fallut toute l'énergie des maîtres du Temple et de l'Hôpital pour dissuader les princes Ferran Sanxe de Castro et Pere Ferrandis de courir sus aux « infidèles », qui multipliaient les provocations dans la plaine d'Acre. Une force de 15000 hommes attendait, en effet, le moindre faux pas des croisés pour fondre sur la capitale par procuration du royaume de Jérusalem ${ }^{43}$. Malgré ces besoins, la détérioration de la conjoncture suscita dans les dernières années du XIII ${ }^{\mathrm{e}}$ siècle des divisions à l'intérieur de l'ordre teutonique, de plus en plus tourné vers l'espace balte. Le grand maître Burchard von Schwanden démissionna au lendemain de son arrivée à Acre, en 1290, pour rejoindre les rangs de l'Hôpital. Il confia au commandeur de Sicile, Heinrich von Bolanden, le soin de défendre la cité aux côtés des autres dignitaires occidentaux. Cette mission avait été rendue urgente par le sac de Tripoli, en avril 1289, au cours duquel un nombre important de templiers et d'hospitaliers avaient trouvé la mort d'après le témoignage du «Templier de Tyr $»^{44}$.

Le même chroniqueur évoque le destin des 14000 combattants à pied et 800 chevaliers ou sergents montés, qui défendirent Acre au printemps $1291^{45}$. Les templiers assurèrent la surveillance de la section la plus occidentale des remparts de la ville au contact des hospitaliers, en charge du secteur courant de la porte de Maupas à celle de Saint-Antoine. Les Acconitains divisèrent leurs effectifs en quatre gardes, permettant d'aligner en permanence la moitié des défenseurs sur les remparts. Le roi de Chypre dirigea la seconde garde avec l'assistance du commandeur Konrad von Feuchtwangen, qui avait succédé à Heinrich von Bolanden, tué prématurément. Les grands maîtres du Temple et de l'Hôpital reçurent le commandement des deux dernières avec l'appui de confréries locales. Ce dispositif permit aux templiers d'effectuer une brillante sortie dans la nuit du 15 avril 1291, sans compromettre la sécurité de la ville ${ }^{46}$.

L'assaut final intervint dans un concert assourdissant de trompes et de tambours, 33 jours plus tard. Les mamelouks parvinrent à isoler la tour Maudite, protégeant la cité, avant de prendre pied sur sa seconde enceinte. Le grand maître du Temple gagna la porte Saint-Antoine, qui menaçait de tomber, avec une douzaine d'hommes et convainquit le maître de l'Hôpital, Jean de Villiers, de tenter de refouler les mamelouks au-delà de la muraille. Le « Templier de Tyr » affirme que les deux hommes se heurtèrent à un «mur de pierre» émergeant des fumées engendrées par les feux grégeois. Guillaume de Beaujeu reçut un dard mortel sous le bras gauche, tandis que Jean de Villiers fut évacué par ses hommes, en état de choc. Les derniers templiers valides s'enfermèrent dans leur couvent fortifié, qui résista

\footnotetext{
${ }^{43}$ Templier de Tyr, op. cit., § 114 (350) p. 114 ; P.-V. Claverie, op. cit. en note 5, t. II, p. 8485.

${ }^{44}$ S. Gouguenheim, Les chevaliers teutoniques, Paris, Tallandier, 2007, p. 265, Templier de Tyr, op. cit., § 239-241 (475-478) p. 196-198.

${ }^{45}$ Ibid., $\S 244-272$ (480-508), p. 198-226.

${ }^{46}$ R.B.C. Huygens, The Fall of Acre 1291 : Excidii Acconis gestorum Collectio; Magister Thadeus civis Neapolitanus, Ystoria de desolatione et conculcatione civitatis Acconenisis et tocius Terre sancte, Turnhout, 2004, p. 59-62 [765-766]; Templier de Tyr, op. cit., § 255 (491) p. 208-210.
} 
une dizaine de jours sous les ordres du maréchal Pierre de Sevrey. Son homologue hospitalier, Matthieu de Clermont, eut une fin non moins héroïque dans le secteur de la porte Saint-Antoine, où il avait réalisé des prodiges par le passé ${ }^{47}$.

La chute d'Acre ne démobilisa pas les ordres militaires qui restructurèrent leurs couvents à la fin de l'année 1291. Templiers et hospitaliers centralisèrent leurs activités à Chypre autour du port de Limassol, regardant vers la Syrie. Ils purent intervenir à partir de cette base opérationnelle en Petite-Arménie avec le soutien ponctuel des teutoniques, repliés en Vénétie. Le pape Nicolas IV leur confia la mission prioritaire de défendre les abords de l'île de Chypre avec une vingtaine de galères. Cette responsabilité conduisit les ordres militaires à participer en 1293 au raid mené contre Candélore par le Génois Manuel Zaccaria à la tête de 25 galères, armées pour les deux tiers par la Couronne chypriote ${ }^{48}$.

Templiers et hospitaliers reprirent du service, six ans plus tard, après la victoire de l'Ilkhan de Perse, Ghazan, sur les mamelouks sous les murs de Homs. Le grand maître de l'ordre teutonique, Gottfried von Hohenlohe, habilita le maréchal ainsi que l'hospitalier de son ordre à prendre part au projet d'alliance échafaudé par les Mongols. Les forces du Temple et de l'Hôpital participèrent au raid naval mené par la marine cypriote jusque dans le delta du Nil, avant de s'établir dans l'îlot de Rouad au cours de l'automne 1300. Les hospitaliers attendirent jusqu'au printemps suivant une jonction avec les Mongols qui ne survint jamais. Ils abandonnèrent Rouad aux templiers après avoir séjourné sur le continent pendant 25 jours. Les mamelouks finirent par s'emparer de l'îlot, le 26 septembre 1302, en capturant 120 chevaliers du Temple esseulés. Cette évidence se heurte au témoignage de certains chroniqueurs arabes, prompts à exagérer l'ampleur de la résistance offerte aux mamelouks à des fins de propagande. Tel est le cas du Syrien Ibn Ka* îr $(† 1373)$ dont la chronique évalue à 2500 hommes l'ampleur de la garnison de Rouad, vaincue en une demi-journée par l'émir Sayf ad-Dīn Zarrāk al-Mansūriì ${ }^{49}$ !

Ces exemples témoignent de la diversité des opérations tactiques entreprises par les ordres militaires à la faveur des croisades. Ces congrégations jouirent pendant deux siècles d'une grande liberté de manœuvre, induite par leur dimension religieuse. Cette indépendance les amena à soutenir fréquemment des options politiques divergentes, tout en coopérant pour le mieux avec les autorités du royaume de Jérusalem. La discipline des frères sauva les armées latines des situations périlleuses où les avaient plongées l'imprudence, l'enthousiasme et

\footnotetext{
${ }^{47}$ Templier de Tyr, op. cit., $\S 262$ (498) p. 216 et 218 ; R.B.C. Huygens, op. cit., p. 70-74 [771-773] et 84-93 [778-783]; J. Richard, Le royaume latin de Jérusalem, Paris, 1953, p. 339-340.

${ }^{48}$ A. Demurger, Jacques de Molay. Le crépuscule des templiers, Paris, 2007 ( $2^{\mathrm{e}}$ éd.), passim, J. Richard, «Le royaume de Chypre et l'embargo sur le commerce avec l'Égypte (fin XIII ${ }^{\mathrm{e}}$ début $\mathrm{XIV}^{\mathrm{e}}$ siècle », Académie des Inscriptions et Belles Lettres. Comptes rendus des séances de l'année 1984, p. 120-134 (repris dans Croisades et Etats latins d'Orient, Aldershot, 1992, $\left.\mathrm{n}^{\circ} \mathrm{XVI}\right)$; P.-V. Claverie, op. cit. en note 5, t. II, p. 242-245.

${ }^{49}$ S. Gouguenheim, op. cit. en note 44, p. 266 et not. 7 p. 668-669; P.-V. Claverie, op. cit., t. II, p. 257-268 ; A. Fuess, Verbranntes Ufer. Auswirkungen mamlukischer Seepolitik auf Beirut und die syro-palästinensische Küste (1250-1517), Leyde, 2001, p. 23.
} 
l'inexpérience des croisés. Chaque ordre était partagé des mêmes lignes de fracture affectant la société franque. Les arrêts émaillant les statuts ou « retraits » du Temple révèlent l'opposition fréquente de combattants invétérés, de penseurs éclairés et de soldats de métier lors de la tenue des chapitres. Les élections magistrales ne profitèrent pas toujours aux hommes les plus avisés, ainsi que les Assassins se plaisaient à le croire au XIII ${ }^{\mathrm{e}}$ siècle. L'élection de personnages excessifs, comme Gérard de Ridefort, ne compromit cependant jamais la solidarité des ordres militaires sur les champs de bataille orientaux. Elle renforça paradoxalement la convergence de vues des frères amenés à trouver la mort sous leurs ordres, à l'instar de Jaquelin de Maillé et Roger des Moulins à La Fontaine du Cresson.

Pierre-Vincent Claverie Assemblée Nationale 